\title{
Steady State and Transient Thermal Analysis of Hot Spots in 3D Stacked ICs using Dedicated Test Chips
}

\author{
H. Oprins, V. Cherman, M. Stucchi, B. Vandevelde, G. Van der Plas, P. Marchal, E. Beyne \\ IMEC \\ Kapeldreef 75, 3001 Leuven, Belgium \\ Herman.Oprins@imec.be
}

\begin{abstract}
3D stacking of dies is a promising technique to allow miniaturization and performance enhancement of electronic systems. The complexity of the interconnection structures, combined with the reduced thermal spreading in the thinned dies and the poorly thermally conductive adhesives complicate the thermal behavior of a stacked die structure. The same dissipation will lead to higher temperatures and a more pronounced temperature peak in a stacked die package compared to a single die package. Therefore, the thermal behavior in a 3D-IC needs to be studied thoroughly. In this paper, a steady state and transient analysis is presented for hot spots in 3D stacked structures. For this analysis, dedicated test chips with integrated heaters and temperature sensors are used to assess the temperature profile in the different tiers of the stack and to investigate the impact of TSVs on the temperature profile. This experimental set-up is used to evaluated and improve the thermal models for the 3D stacks.
\end{abstract}

\section{Keywords}

3D integration, thermal characterization, through-Si vias (TSVs), thermal modeling.

\section{Introduction}

3D stacking of chips is a promising technique to allow miniaturization and performance enhancement through the reduction of interconnect lengths in electronic systems [1]. Thermal management issues in the 3D stacks are considered to be one of the main challenges for 3D integrations $[2,3]$. The use of poorly thermally conductive adhesives, the vertical integrations of the chips and the reduced thermal spreading due the aggressively thinned dies cause these thermal management issues. In the case of hot spots, these thermal effects are even more pronounced $[9,10]$. As a result, the same power dissipation in a 3D stack will lead to higher temperatures and more pronounced temperature peaks in a stacked die package compared to a single die package. To address the thermal issues of $3 \mathrm{D}$ integration, several advanced dedicated cooling solutions are developed ranging from thermal herding by smart placement of thermal vias [4] over conduction-convection based cooling solutions [5] to complex inter-tier liquid cooling solutions $[6,7,8]$. The interconnection structures, back end of line structures and through-Si vias (TSVs) complicate the thermal behavior of a stacked die structure increase the complexity of the conductive heat transfer paths in a stacked die structure. To understand the effect of these TSVs and inter-tier connections, a detailed analysis of the heat transfer in 3D stacks, including the TSVs is needed. [9] describes the estimation of the thermal resistance of the interconnection for the 3D integration using a combination of modeling and experimental study.

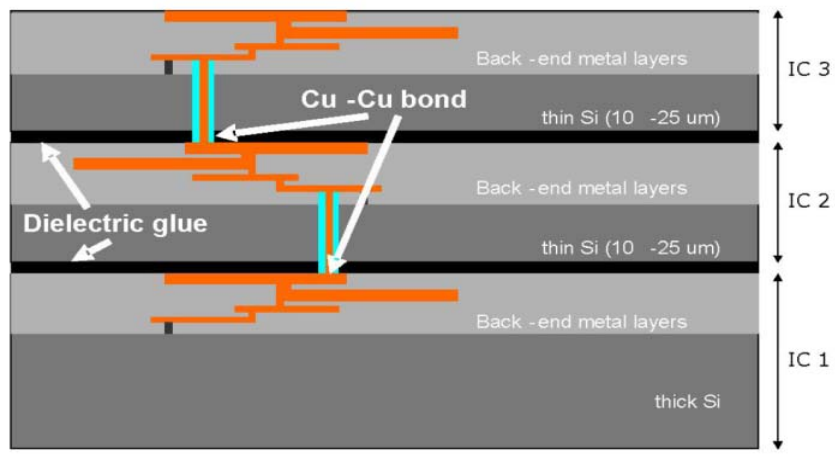

Figure 1. Schematic cross section of the 3D integration using through Silicon vias [13].

In this paper, the thermal behavior of the hot spot in stacks and the impact of TSVs on the temperature profile are studied using dedicated thermal test chips with integrated heaters and diodes. Stacks consisting of theses test chips are used to evaluate thermal modeling methodologies for $3 \mathrm{D}$ integration and to explore the effect of possible cooling solutions. The experimental results allow to calibrate the thermal models and to assess the thermal impact of the interconnection technology used for the 3D integration.

\section{Thermal test vehicle for stacked die structures}

To characterize the thermal behavior in the 3D stack, thermal test chips are used. Such a thermal test chip mimics the power dissipation of a real chip and at the same time measures the temperature at different locations in the die stack. In this work 3D stacked ICs using through-silicon vias (TSVs) are considered. In this approach the connection is achieved by copper to copper bonding. A schematic of the 3D-SIC architecture is shown in Figure 1 and an in depth description of the 3D-SIC process can be found in $[12,13]$. The $5 \mathrm{~mm} \times 5 \mathrm{~mm}$ top die is thinned down to $25 \mu \mathrm{m}$ and connected using copper to copper bonding to a full thickness 8 $\mathrm{mm}$ by $8 \mathrm{~mm}$ die. Figure 2 shows an overlay of the design of the top and bottom die of the stack, with an indication of the integrated test structures.

\subsection{Test chip with integrated heaters and sensors}

To dissipate power, $\mathrm{Cu}$ meander resistor heaters in the metal 2 layer (M2) of the Back End (BEOL) of the top die are used. Heater sizes of $50 \times 50$ and $100 \times 100 \mu \mathrm{m}^{2}$ are used to study the thermal effect of the hot spot size. The electrical resistance of the meander heaters is $3400 \pm 750 \Omega$ and $800 \pm 18$ 
$\Omega$ for the $100 \times 100 \mu \mathrm{m}^{2}$ and $50 \times 50 \mu \mathrm{m}^{2}$ respectively. The metal layers of the BEOL are separated from the Si of the die by an oxide with a low thermal conductivity. Therefore a large temperature drop will occur between the heater and the $\mathrm{Si}$ of the test die. The reported temperatures are the temperatures in the $\mathrm{Si}$. The temperatures in the heaters are significantly higher. Diodes in both top and bottom die are used as temperature sensors. A constant diode current of 10 $\mu \mathrm{A}$ is used for the diode measurements since in this case the power dissipation of the diode itself is negligible compared to the power in the die and self-heating in the diode is avoided. In this way, the die temperature is not affected by the diode measurement. At the position of each heater, a set of 5 diodes at different distances $(0,60,80,120$ and $160 \mu \mathrm{m}$ respectively) from the hot spot centre are added to capture the local temperature peak. This allows for characterization of the narrow temperature peak at the hot spot and of the effect of hot spot size and interface layers on the peak shape. The position of diodes in the top die overlaps with diodes in the bottom to study the vertical heat conduction in the stack. This can be used to characterize the thermal properties of the polymer glue layer between the top and bottom die. With this set of diodes and heaters both the horizontal and vertical heat spreading in the stack can be accurately studied.

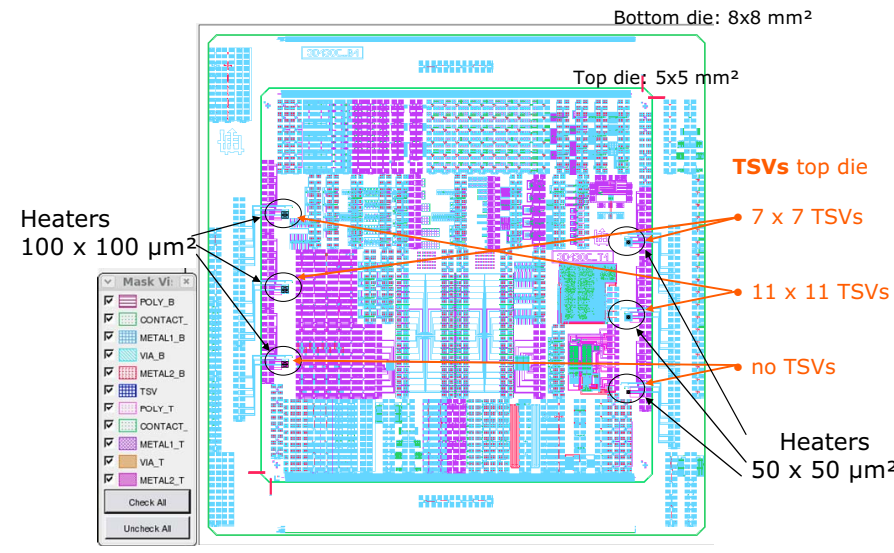

Figure 2. Design layout of the stack of the thermal test chip with the thermal test structures.
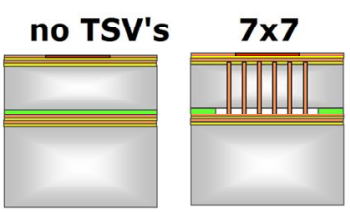

$11 \times 11$

(a)

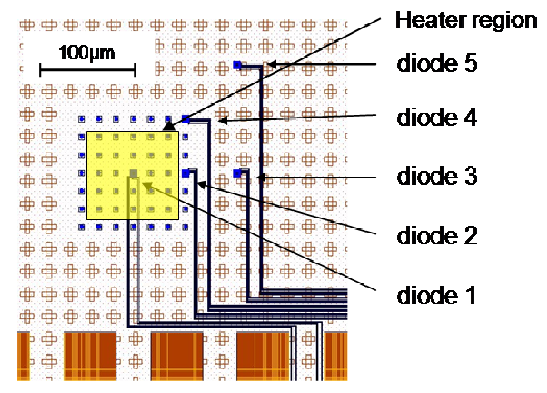

(b)
Figure 3. (a) Schematic cross-section of the die stack at the location of the test structures, revealing the TSV array density. (b) Detail of the design layout showing the heater, diodes and TSV array.

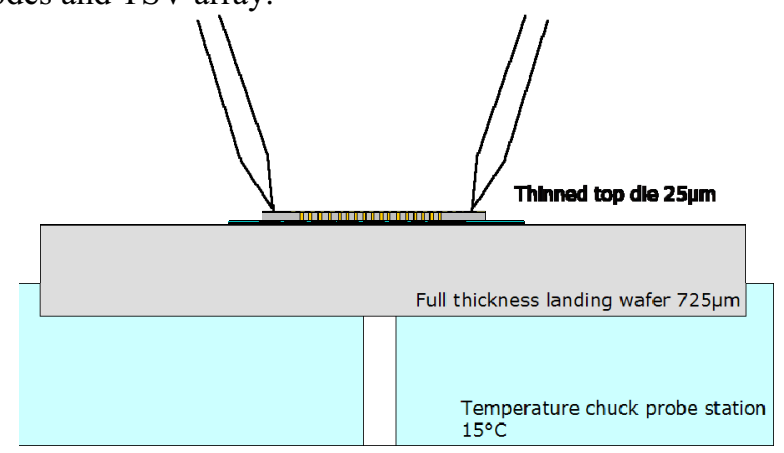

Figure 4. Schematic cross-section of the measurement set-up; the two die stack is mounted on a temperature controlled chuck and kept in place using vacuum.

Different TSV densities are used in the die locally below the heaters to characterize their ability to locally enhance the heat transfer: a reference structure without TSVs, an array of $7 \times 7$ TSVs (5\%) and 12x12 TSVs (12\%) are used. The TSVs are connected to the bottom die using $\mathrm{Cu}-\mathrm{Cu}$ bonding. Figure 2 gives an overview of the location of the 6 test structures $(2$ heater sizes, $3 \mathrm{TSV}$ densities) that are implemented on the top die. Figure 3(a) gives a schematic representation of the crosssection of the test structures. In Figure 3(b), a detail of the layout at the location of a test structure is shown to reveal the location of the heater, diodes and TSV array.

\subsection{Experimental set-up}

To be able to evaluate the thermal modeling early in the development of the 3D integration including the TSVs, a more simplified version of the packaged die stack is considered. In the thermal analysis described in this paper, the thermal measurements are performed on a wafer level probe station. A thinned top die ( $25 \mu \mathrm{m}$ thickness) is bonded to a full thickness landing wafer $(725 \mu \mathrm{m})$. The die stacked is mounted on a chuck and kept in place by vacuum. The temperature of this chuck can be controlled to provide an isothermal boundary to the bottom of the die stack. This set-up is schematically shown in Figure 4. Probes assessing the bond pads of the top die are used to electrically connect to the heaters and diodes to dissipate heat in the meander heaters and measure the voltage of the diode for the constant current of $10 \mu \mathrm{A}$. The distance between bond pads and diode is sufficient not to interfere with the thermal measurements. If the bond pads and the probes are too close to the diodes they might act as a local heat sink and influence measured temperatures. In this case the distance is more than $1 \mathrm{~mm}$; therefore it can be considered that the probes will not have an influence on the local temperature profile close to the heater $\left(50 \times 50 \mu \mathrm{m}^{2}\right.$ or $\left.100 \times 100 \mu \mathrm{m}^{2}\right)$.

\section{Modeling approaches for thermal simulation of 3DICs}

Different thermal modeling approaches for 3D-ICs can be used for different stages in the design process. This ranges from a fast solving simplified calculation tool for the temperature distribution in the different chips of the stack to a 
detailed fine grain simulation including all layout details for the final verification of the design.

In [14], a thermal compact model for 3D stacked structures has been presented. This compact model has been developed to implement the thermal-aware floor-planning in 3D stacks in the early stages of the design. With this model, the temperature distribution is calculated in each die, using the power maps of the heat generation in each tier as input. This compact model allows studying the thermal interaction of heat sources in the 3D stack, both on the same die as well as on other levels of the stack. Furthermore, the compact model allows thermal optimization of the placement of the heat sources as a function of the geometrical and material properties of the interface and interconnects structures. In Figure 5(a), the graphical interface of this compact model is shown.

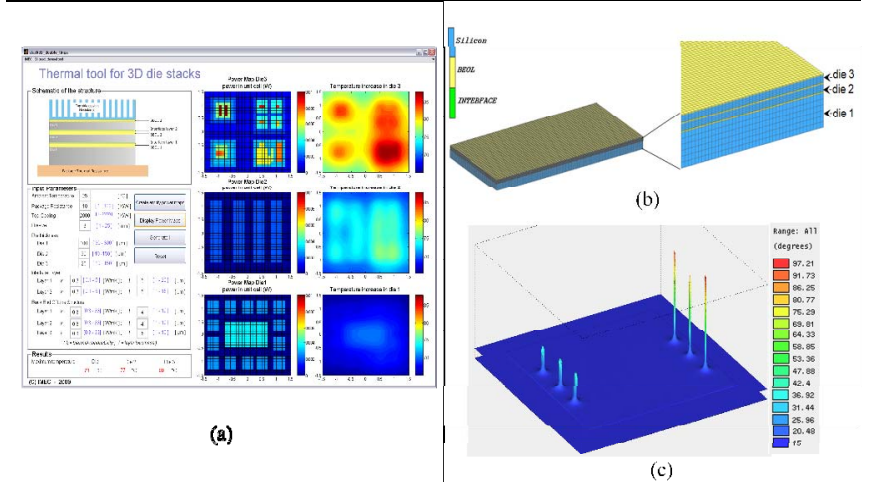

Figure 5. Thermal modeling approaches for $3 \mathrm{D}$ stacks with increasing complexity and accuracy; (a) compact thermal model [14], (b) package level finite element modeling [16], (c) fine grain thermal modeling [15].

In [15], a modeling methodology to perform a fine grain thermal simulation of $3 \mathrm{D}$ stacks has been presented. The geometry input for this simulation is based on the complete design layout (GDS II files) of all the dies in the stack. This approach can be used for the final thermal verification and the sign-off of the design. Figure 5(c) shows an example of the detailed thermal simulations in a 3D stack. In this figure, a surface plot of the temperature distribution in the top die is shown.

An intermediate finite element modeling (FEM) approach has been presented in [16]. This FEM approach provides a higher level simulation, compared to the fine grain thermal simulation, by using average material properties to replace the complicated interconnection and Back End structures. This allows including the presence and effect of these structures in the simulation but still results in a reasonable calculation time. As a result this approach can be used for optimization and transient thermal analysis of the 3D stack. In this paper, the results of this FEM approach are compared to the experimental results obtained using the presented test vehicle.

\section{Steady state thermal analysis}

In this section, the steady state thermal measurement results are discussed and compared to the modeling results obtained using the FEM approach discussed in the previous section. The diode temperatures are measured for 10 different stacks. In the following graphs, the average of the 10 Oprins, Thermal analysis of hot spots in 3D stacked ICs ... measurements will be shown, accompanied by the $95 \%$ confidence interval.

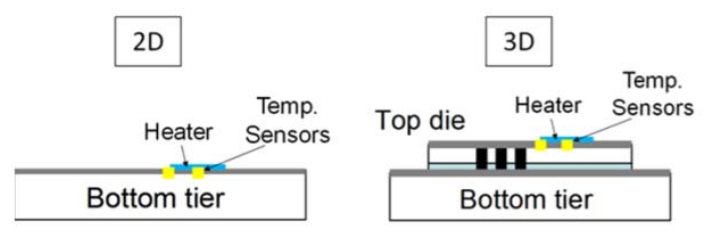

(a)

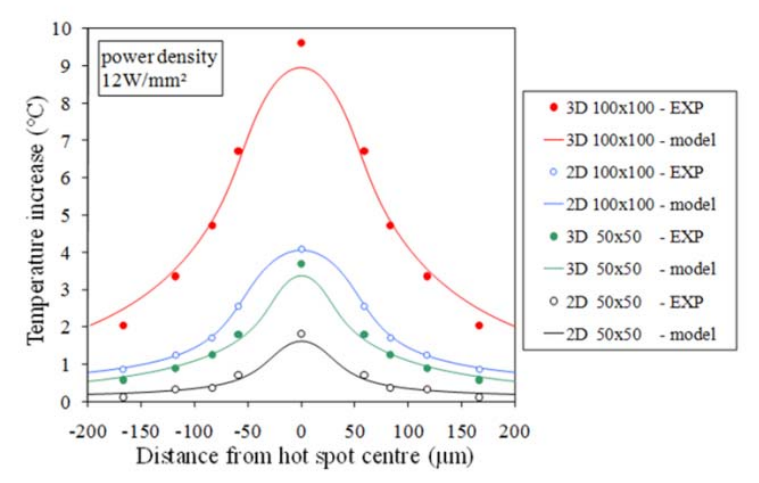

(b)

Figure 6. (a) Schematic representation of the $2 \mathrm{D}$ and stacked 3D configuration. (b) Temperature profile simulation (solid lines) and experimental (markers) results for a power dissipation of $12 \mathrm{~W} / \mathrm{mm} 2$ in $100 \times 100 \mu \mathrm{m} 2$ and $50 \times 50 \mu \mathrm{m} 2$ heaters in the $2 \mathrm{D}$ and $3 \mathrm{D}$ configuration.

\subsection{Comparison of $2 \mathrm{D}$ vs. $3 \mathrm{D}$ integration}

First, the temperature profile for hot spot dissipation is compared for a 2D reference case and for the top die of a 3D stack. The 2D reference case is a full thickness single die with the integrated heaters and temperature sensors. In the 3D case, the heater is in the thinned top die, stacked on top of a full thickness bottom die. Figure 6(a) shows a schematic representation of the $2 \mathrm{D}$ and $3 \mathrm{D}$ configuration. The temperature effect is compared for both the $100 \mu \mathrm{m} \times 100 \mu \mathrm{m}$ as the $50 \mu \mathrm{m} \times 50$ heater, using a power density of $12 \mathrm{~W} / \mathrm{mm} 2$ in the hot spot. This amounts to a power dissipation of 120 $\mathrm{mW}$ and $30 \mathrm{~mW}$ in the large heater and the smaller heater respectively. Figure 6(b) shows the experimental and modeling results for the temperature profiles in case of the test structure without TSVs. This graph indicates that the local temperature increase in the case of the $3 \mathrm{D}$ configuration is three times as high compared to the $2 \mathrm{D}$ reference case both for the $100 \mu \mathrm{m} \times 100 \mu \mathrm{m}$ as well as the $50 \mu \mathrm{m} \times 50 \mu \mathrm{m}$ heater. Besides an increase in peak temperature, also a broadening of the temperature peak can be observed.

The measurement results indicate that the diodes are capable of capturing the shape of the local temperature peak below the hot spot, both for the large and the small heaters. Furthermore, from this figure, a very good agreement between the modeling and experimental temperature measurements can be observed.

\subsection{Influence of hot spot size and TSV density}

Figure 7 shows the normalized temperature profiles in the top die for the test structures with the $100 \mu \mathrm{m} \times 100 \mu \mathrm{m}$ heater (Figure 7a) and the $50 \mu \mathrm{m} \times 50$ heater (Figure 7b). For 
the experimental measurements, the $95 \%$ confidence intervals based on 10 different stacks are shown as error bars in the graphs. In these graphs, the experimental results are compared to the modeling results. For the $100 \times 100 \mu \mathrm{m} 2$ heater the normalized peak temperature is $82 \mathrm{~K} / \mathrm{W}$. In case of the $50 \times 50$ $\mu \mathrm{m}^{2}$ heater, the normalized peak temperature is $125 \mathrm{~K} / \mathrm{W}$. In the case of the test structures without TSVs, the agreement between the simulation and experimental results is very good. Not only is the temperature peak predicted very accurately by the modeling, but also the shape of the temperature profile matches nicely with the temperature measurements in the diodes. For the whole profile the modeling results are within the range of the $95 \%$ confidence interval of the experiments. The maximum differences between the modeling and the average value of the experiments are less than $5 \%$ for the temperature peaks and less than $10 \%$ in the tail.

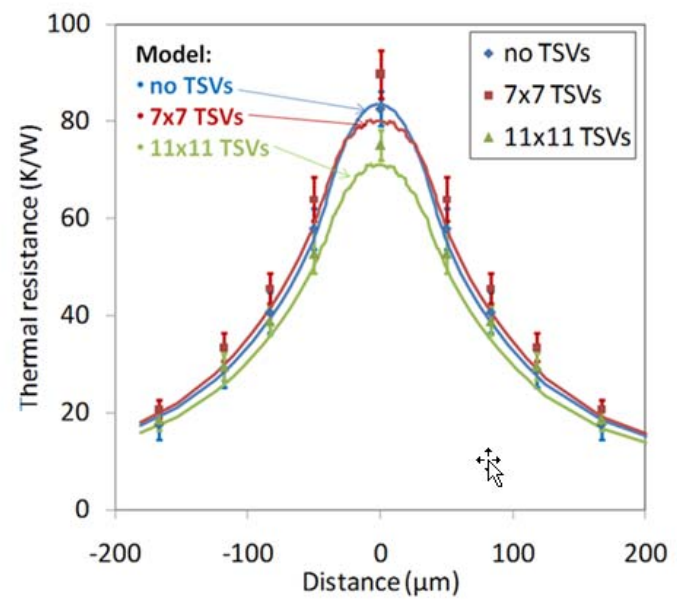

(a)

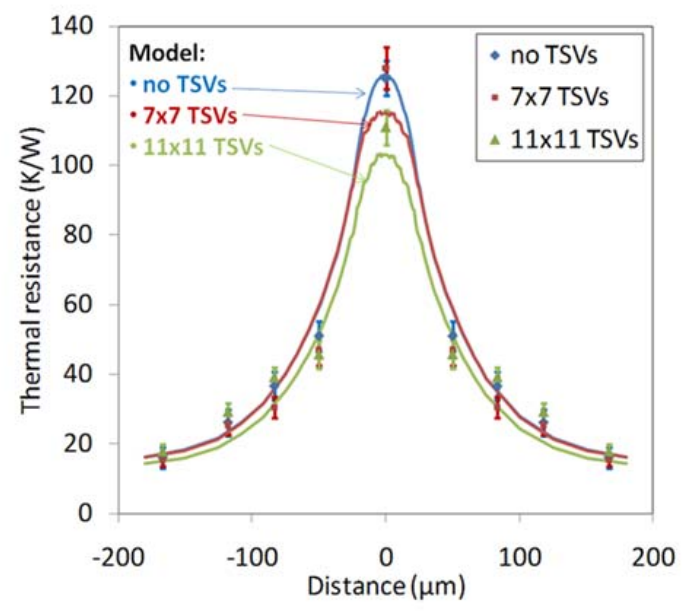

(b)

Figure 7. Results of the initial thermal simulation (solid lines) and experimental measurements (markers and error bars) using the test vehicle for the different TSV densities for the $100 \times 100 \mu \mathrm{m}^{2}$ heater (a) and $50 \times 50 \mu \mathrm{m}^{2}$ heater (b).

In the case of the presence of TSVs below the test structures, a discrepancy between the experimental and the simulation results can be observed: the simulations predict a decrease in peak temperature for an increasing TSV density (no TSVs $<7 \times 7$ TSVs $<11 \times 11$ TSVs), however experimentally a temperature increase has been observed for all 10 test die, from the reference case (no TSVs) to the case with an array of $7 \times 7$ TSVs. On the other hand, the relative temperature decrease $(-20 \%)$ for an increasing TSV density from $7 \times 7 \mathrm{TSVs}$ to $11 \times 11 \mathrm{TSVs}$ is correctly predicted by the simulations. This indicates that certain processing variability related phenomena are not included in the model to correctly predict the thermal impact of TSVs and inter-tier connections and that the model should be updated.

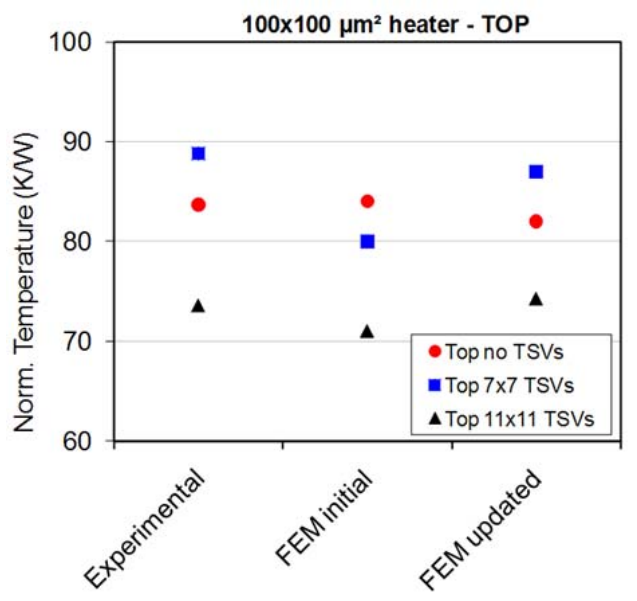

(a)

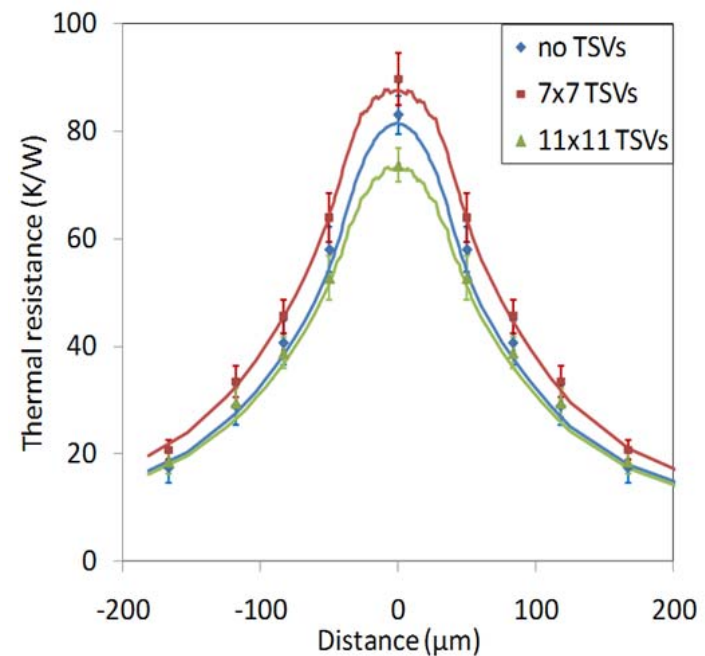

(b)

Figure 8. Calibration for the $100 \times 100 \mu \mathrm{m}^{2}$ heater: (a) Peak temperatures for the initial modeling results, the experimental results and the updated finite element modeling results. (b) Comparison of the temperature profiles simulated by the updated model to the experimental results.

\subsection{Model calibration}

To improve the model, a detailed Focused Ion Beam analysis (FIB) has been performed on the cross-section of the stack. This detailed FIB analysis reveals the presence of phenomena that can cause the discrepancy between the experimental and modeling results. Using this analysis, slight variations in the stand-off height between the top and bottom die and a non-uniform distribution of the polymer adhesive can be observed. Furthermore, it is observed that the outer 
ring of the TSV array of the top die is not everywhere connected to the bottom die. This observation is in line with the electrical resistance measurements of the TSVs which were performed to assess the landing of the TSVs [17]. The finite element model has been updated taken into account these deviations from the designed geometry. Figure 8a shows the impact of those changes to the simulation results for the different TSV densities. In Figure 8a, the peak temperatures are shown for the initial modeling results, the experimental results and the results from the updated model. Figure $8 \mathrm{~b}$ shows the temperature profiles extracted from the updated model, compared to the measured diode temperatures. From this graph can be seen that the updated model is able to predict the correct trend of the impact of TSVs on the temperature profile of the top die. This analysis indicates that it is important to take the process variations into account when assessing the temperature profile and the impact of TSVs in 3D stacks. In the case of $\mathrm{Cu}-\mathrm{Cu}$ bonding of the TSVs with a stand-off height of $700 \mathrm{~nm}$ to $1 \mu \mathrm{m}$, the impact of the process variations is of the same order as the impact of the TSVs on the temperature profile.

\section{Transient thermal analysis}

In this section, the transient temperature response of the top and bottom die is discussed for hot spot dissipation in the top die. Figure 9 shows the evolution of the diode temperature in the five diodes for both the top and bottom die for the structure without TSVs with a power dissipation of $480 \mathrm{~mW}$ in the $100 \mu \mathrm{m} \times 100 \mu \mathrm{m}$ heater. The location of the five diodes in the region of the heater is shown in Figure 3b. The temperature of the diodes has been measured using a time resolution of $10 \mu \mathrm{s}$. This resolution is sufficient to capture the fast temperature rise for the hot spot dissipation. In Figure 10, the temperature profile is shown at different time steps: $10 \mu \mathrm{s}$, $40 \mu \mathrm{s}, 100 \mu \mathrm{s}, 400 \mu \mathrm{s}$ and $1 \mathrm{~ms}$. These subsequent profiles illustrate the temperature rise and the broadening of the temperature peak in time. It can be observed that temperature response of the hot spot dissipation reaches a steady state condition around $2 \mathrm{~m} \mathrm{~s}$. From $2 \mathrm{~m} \mathrm{~s}$ on, the effect of the heating of the package can be observed. This measurement illustrates that the transients in case of hot spots are much faster compared to uniform heating. In the case of uniform heating in the top die of a stack, a time constant of the order of $1 \mathrm{~s}$ is typically found [18].

In Figure 9 and 10, the experimental diode temperature measurements are compared to the transient results of the update finite elements model. The comparison between the modeling and experiments shows a good agreement over the whole range of time scales. From these measurements, the time constants of the temperature response can be extracted. It is found that the time constants for the temperature response are $100 \mu \mathrm{s}$ and $200 \mu \mathrm{s}$ for the top and bottom die respectively. Figure 11 shows the normalized temperature response of the top and bottom die, normalized with respect to their respective temperature at $10 \mathrm{~ms}$. This graph illustrates the thermal delay between the top and the bottom die.

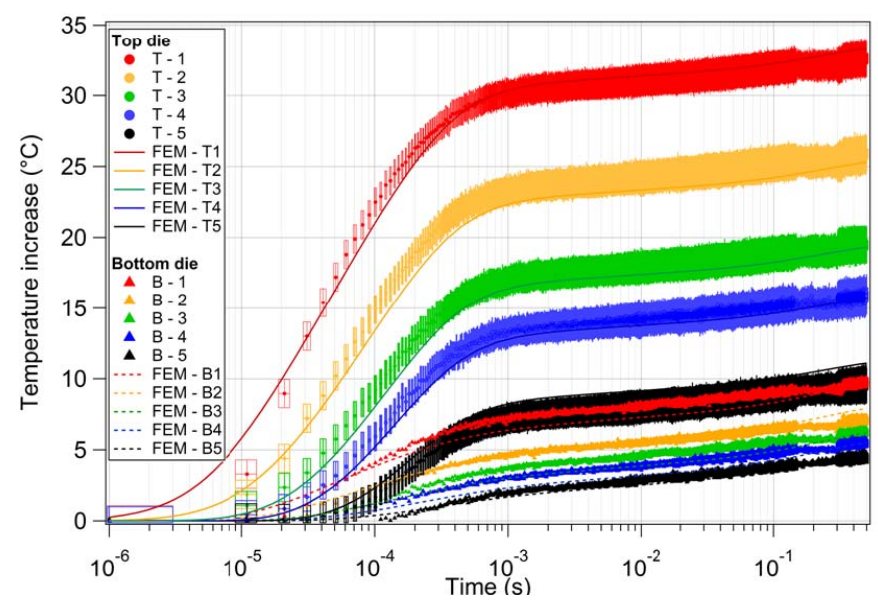

Figure 9. Transient evolution of the temperatures at the locations of the diodes shown in Figure 3 in both the top and bottom die of the stack. The solid lines represent the simulation results of the updated FE model. The experimental results are shown by the markers and error bars.

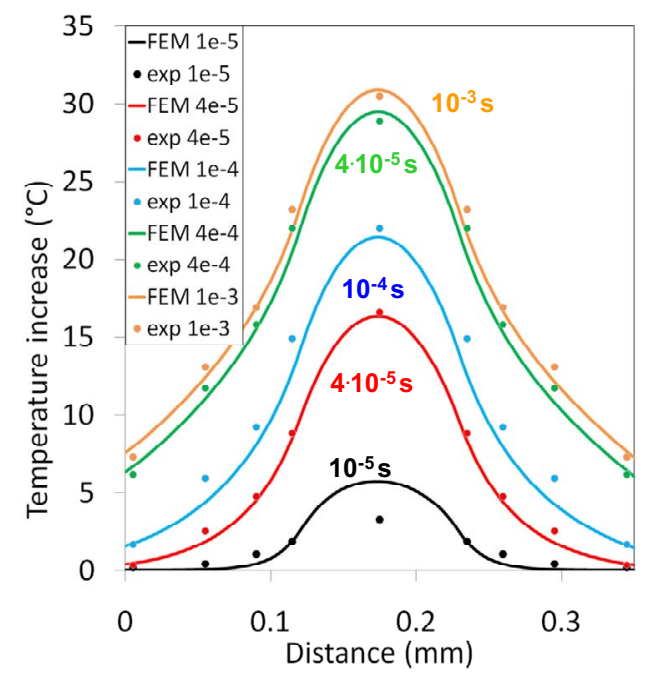

Figure 10. Temperature profile (experimental and numerical) in the $\mathrm{Si}$ below the $100 \times 100 \mu \mathrm{m}^{2}$ hot spot in the top die at different time steps.

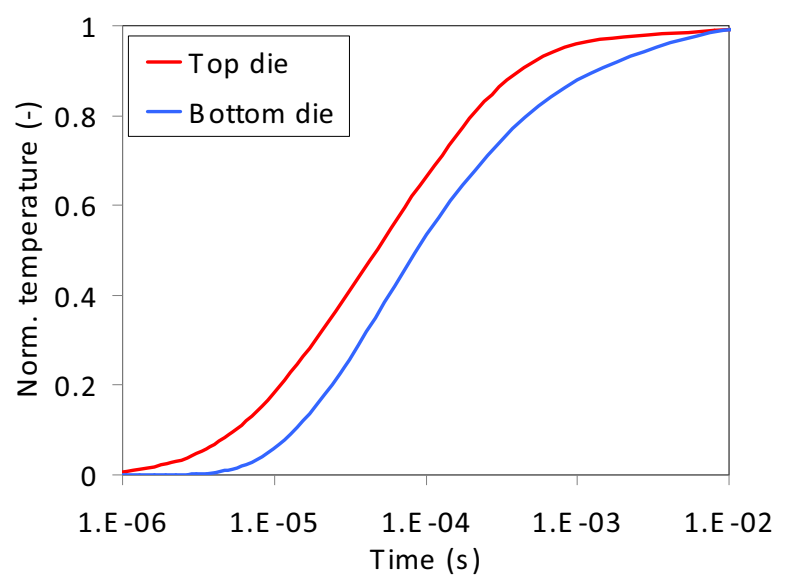

Figure 11. Normalized temperature response of the temperature in the top and bottom die for a power dissipation in the top die. 

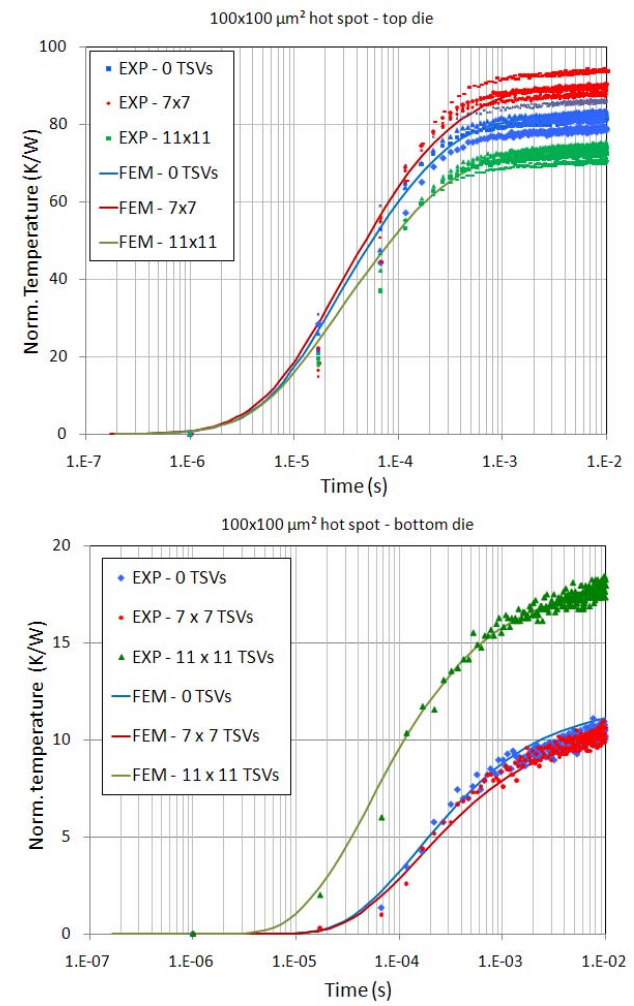

Figure 12. Transient evolution of the peak temperatures (diode 1) for the different TSV densities for the top and bottom die in case of the $100 \mu \mathrm{m} \times 100 \mu \mathrm{m}$ hot spot. The experimental results (markers: $\cdot,-, \mathbf{m}, \diamond$ ) are shown for 4 different test chips.
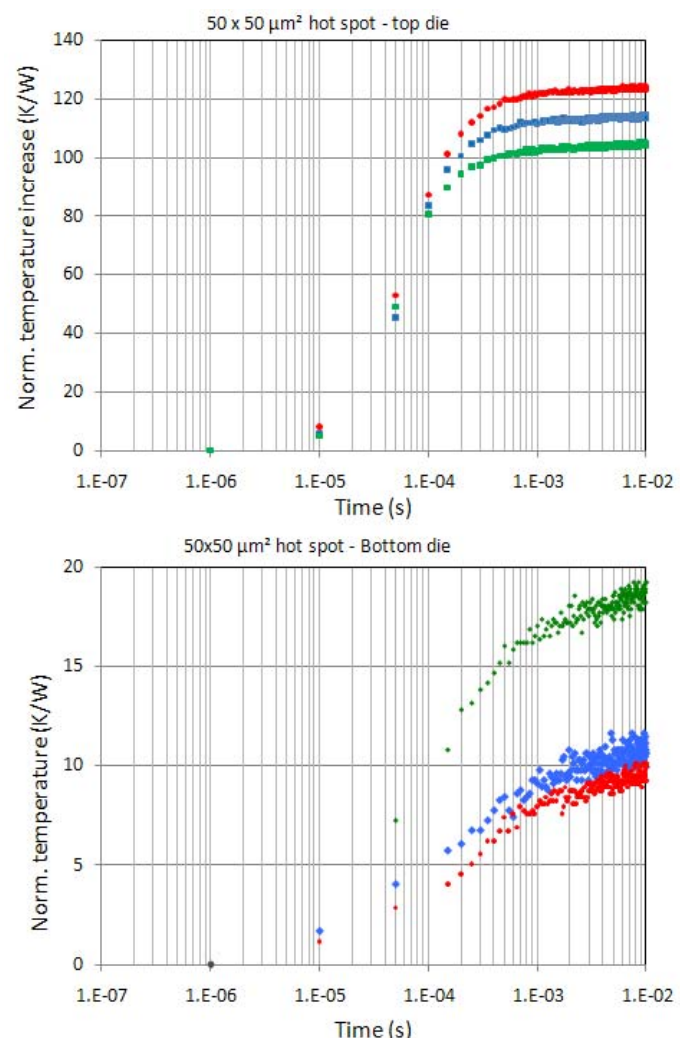

Figure 13. Transient evolution of the peak temperature (diode 1) for the different TSV densities for the top and bottom die in case of the $50 \mu \mathrm{m} \times 50 \mu \mathrm{m}$ hot spot..
Figure 12 and 13 show the comparison between the modeling (solid lines) and the experimental results (markers) for the peak temperatures in the cases of the different TSV densities for the $100 \mu \mathrm{m} \times 100 \mu \mathrm{m}$ and the $50 \mu \mathrm{m} \times 50 \mu \mathrm{m}$ hot spots respectively. The case without TSVs is the reference case. In the case of $7 \times 7$ TSVs, a reduced heat transfer through the top die is observed. Since the heat is removed through the bottom of the stack, this results in a higher temperature of the top die, and a lower temperature of the bottom die. In this case, the negative effect of the process variations dominates the positive effect of the added vertical connection with high thermal conductivity. In the case of the $11 \times 11$ array of TSVs, the positive effect of the high density array of TSVs is larger than the negative impact of the nonconnected TSVs. As a result, an enhanced vertical heat transfer through the stack is observed: in the case of the 11 $\mathrm{x} 11 \mathrm{TSV}$ array, the temperature of the top die is lower and the temperature of the bottom die is higher compared to the reference case.

\section{Conclusions}

In this paper, a steady state and transient analysis of the temperature profile in 3D stacked structures is presented. For the analysis, a stack of dedicated test chips with integrated meander metal heaters and arrays of temperatures sensing diodes, in the region of the heaters, have been used. On the test chip, test structures with different hot spot size $(50 \mu \mathrm{m}$ and $100 \mu \mathrm{m})$ and different TSVs are present. The set of five diodes below each heater is capable of capturing the steady state and transient profile of the temperature peak of the hot spot dissipation for both the large and the small hot spot. The transient response of the hot spot dissipation is very fast compared to uniform dissipation. A time resolution of $10 \mu \mathrm{s}$ for the transient measurements allows capturing the full transient profile of the heating curve.

The dedicated test chips are used to validate the thermal modeling of the $3 \mathrm{D}$ stack. A good agreement between the modeling and the experiments is found in the case without TSVs. The maximum error is less than $5 \%$ in the temperature peak and less than $10 \%$ in the tails of the temperature profile. For the case with TSVs, a discrepancy is found between the simulation and the experimental results. Using the results of a detailed FIB analysis on the cross-section of the test chips, an updated more, accurate simulation model is constructed. The updated model accurately predicts the impact of the density of the TSV array on the temperature profile both for the steady state and transient analysis. From the transient analysis, a time constant of $100 \mu \mathrm{s}$ is obtained for the temperature response of the die for the hot spot dissipation.

\section{References}

[1] Beyne, E. "The Rise of the 3rd Dimension for System Integration”, Proc. IEEE IITC, pp. 1-5, 2006.

[2] JEDEC Standard, JEP-158 3D Chip Stack with ThroughSilicon Vias (TSVS): Identifying, Evaluating and Understanding Reliability Interactions, www.jedec.org

[3] Sapatnekar, S.S. "Addressing thermal and power delivery bottlenecks in 3D circuits", Proceedings of the 2009 Asia and South Pacific Design Automation Conference 2009. 
[4] Cong, J. ; Wei, ,J.; Zhang, Y., "A thermal-driven floorplanning algorithm for 3D ICs," ICCAD 2004, pp.306-313.

[5] Kota, K.; Hidalgo, P.; Joshi, Y.; Glezer, A., "A novel conduction-convection based cooling solution for $3 \mathrm{D}$ stacked electronics”, SEMI-THERM 2010, pp 33-40.

[6] B. Dang, M. Bakir, D. Sekar, and J. Meindl, "Single and 3D chip cooling using microchannels and microfluidic chip input/output (I/O) interconnects," IEEE Trans. Adv. Packaging, vol. 3, no. 1, pp. 79-87, Feb. 2010.

[7] T. Brunschwiler and B. Michel, "Thermal Management of Vertically Integrated Packages," in Handbook of 3D Integration: Technology and Applications of 3D Integrated Circuits, edited by P. Garrou, C. Bower and P. Ramm (Wiley-VCH Verlag GmbH, Weinheim, 2008) Vol. 2, Part IV, pp. 635-649.

[8] Koo, J.M., Im, S., Jiang, L., and Goodson, K.E., 2005, "Integrated Microchannel Cooling for ThreeDimensional Circuit Architectures," ASME Journal of Heat Transfer, Vol. 127, pp. 49-58.

[9] Rencz, M.; Székely V.; "Structure function evaluation of stacked dies", Proceedings of the XXth SEMI-THERM Symposium, March 9-11, San Jose, CA, USA, pp 50-55, 2004.

[10] Zhang, L.; Howard, N.; Gumaste, V.; Poddar, A.; Nguyen, L.; "Thermal Characterization of Stacked-Die Packages", 20th IEEE SEMI-THERM Symposium, pp. 55-63, 2004.

[11] Matsumoto K., Taira Y.: "Thermal resistance measurement of interconnection, for the investigation of the thermal resistance of a three-dimensional (3D) chip stack" , Semi Therm 2009 (25th Semiconductor Thermal
Measurement and Management Symposium), pp. 321 328, 2009.

[12] Swinnen, B.; Ruythooren, W.; De Moor, P.; Bogaerts, L.; Carbonell, L.; De Munck, K. ; Eyckens, B.; Stoukatch, S. ; Sabuncuoglu Tezcan, D.; Tokei, Z.; Vaes, J. ; Van Aelst, J. and Beyne, E.; "3D integration by Cu$\mathrm{Cu}$ thermocompression bonding of extremely thinned bulk Si die containing $10 \mu \mathrm{m}$ pitch through Si vias", Proc. IEDM Conference, December 11-13, 2006, San Fransisco.

[13] Swinnen, B.; Jourdain, A. ; De Moor, P. ; Beyne E.; Chapter in Wafer Level 3-D ICs Process Technology; edited by S. Tan, R.J. Gutmann, and L.R. Reif (Eds), Springer, ISBN 978-0-387-76532-7, 2008.

[14] Torregiani, C.; Oprins, H.; Vandevelde, B.; Beyne, E.; De Wolf, I.;" Compact thermal modeling of hot spots in advanced 3D-stacked structures", Proc. EPTC 2009, pp. 131-136.

[15] Oprins H. et al., "Fine grain thermal modeling and experimental validation of 3D-ICs", Microelectronics Journal, 2010, in press.

[16] Torregiani, C.; Oprins, H.; Vandevelde, B.; Beyne, E.; De Wolf, I.; "Thermal analysis of hot spots in advanced 3D-stacked structures", THERMINIC 2009.

[17] Van der Plas, G; et al.," Design issues and considerations for low-cost 3D TSV IC technology", ISSCC 2010, pp.148-149.

[18] H. Oprins, V. Cherman, C. Torregiani, M. Stucchi, B. Vandevelde and E. Beyne, Thermal test vehicle for the validation of thermal modelling of hot spot dissipation in 3D stacked ICs, ESTC2010, September 2010. 